\title{
Long-term unilateral visual cortical lesions and learning of a black-white discrimination by one-eyed rats ${ }^{1)}$
}

\author{
Yukinobu IKEDA \\ Department of Psychology, Faculty of Culture and Education, Saga University, \\ 1 Honjyo, Saga 840-8502, Japan, \\ Makoto SAKAI \\ Neuropsychology Laboratory, Saga Medical School, \\ Nabeshima, Saga 849-8501, Japan, \\ and \\ Fumio YAGI \\ Neuropsychology Laboratory, Kochi Medical School, \\ Nankoku, Kochi 783-8505, Japan
}

In a previous study we investigated the effects of callosal transected lesions made 10 weeks earlier (the 10-week-old callosal transected lesions), either at 3 weeks of age or at 13 weeks of age, upon the acquisition of a black-white (BW) discrimination in rats either with one eye removed at birth (OEB) or at 13 weeks of age (OET) following lesions of the contralateral (CT) visual cortex to the remaining eye. The CT visual cortex lesions were performed right before the training of BW discrimination. We found that the 10 -week-old callosal transected lesions facilitated the acquisition in OEBs when the callosal lesions were given at 3 weeks of age, and to a lesser extent at 13 weeks of age. We also found that the same type of callosal transected lesions did not do so in OETs, regardless of the age when the callosal lesions were made.

Since the CT visual cortex lesions inevitably result in damage of the callosal neurons, and lead to degeneration of the callosal afferents in the ipsilateral (IP) visual cortex, the present study was undertaken to investigate if the CT visual cortex lesions made 10 weeks earlier (the 10-week-old CT visual cortex lesions), made either at 3 weeks of age or 13 weeks of age, would affect the acquisition of BW discrimination in OEBs and OETs, like the 10-week-old callosal transected lesions employed in the previous study mentioned above.

We found that in both OEBs and OETs the overall pattern of the facilitation effects of the 10-week-old CT visual cortex lesions on the acquisition of BW discrimination is, in general, the same as that observed in the 10-week-old callosal transected lesions, but the facilitative effect of the 10-week-old CT visual cortex lesions is more prevailing and pronounced. The findings are discussed in relation to the possible involvement of neurotrophic factors, released when the CT visual cortex lesions were made, in synaptic reorganization, and also in relation to the possibility of the increased use of the uncrossed visual pathways.

Key words: Unilateral visual cortical lesion, Monocular enucleation at birth, Black-white discrimination, Facilitation of learning, Albino rat.

1) This investigation was supported in part by a Grant-in-Aid for Scientific Research (C) (No.09610085) from the Ministry of Education, Science, Sports and Culture of Japan.

Received Aug. 4, 1999; Accepted Feb. 4, 2000 


\section{Introduction}

It is well known that monocular enucleation at birth or in early postnatal days results in expanded uncrossed visual pathways to the visual cortex ipsilateral to the remaining eye (IP visual cortex), via the dorsal lateral geniculate nucleus (LGNd), and via the superior colliculus (SC) in rats (e.g., Chan \& Jen, 1988; Jeffery, 1985; Jen \& Lund, 1981; Laemle \& Labriola, 1982; Lund, Cunningham, \& Lund, 1973, Sefton \& Lam, 1984). Behaviorally, 3-month-old rats with one eye removed at birth (OEBs) can relearn a black-white (BW) discrimination faster than those rats with one eye removed at 13 weeks of age (OETs), when relearning is initiated 10 days after lesions of the visual cortex contralateral to the remaining eye (CT visual cortex). In this case the CT visual cortex lesions are made the day following the completion of original learning (Ikeda, Sakai, \& Yagi,1988; Ikeda, Sakai, \& Yagi, 1992; Ikeda, Yagi, \& Sakai, 1983; Sakai, Ikeda, \& Yagi, 1991; Sakai, Ikeda, \& Yagi, 1996; Yagi \& Sakai, 1979; Yagi, Sakai, \& Ikeda, 1989). This "faster" relearning phenomenon of OEBs is considered to be a behavioral expression of the above mentioned reorganization in the visual system.

However, our previous studies (Ikeda et al., 1988; Ikeda et al., 1992; Ikeda et al., 1983; Sakai et al., 1991; Sakai et al., 1996; Yagi \& Sakai, 1979; Yagi et al., 1989) have also demonstrated that neither OEBs nor OETs are able to master BW discrimination when the discrimination training is given at 3 months of age and made 10 days after damage of the CT visual cortex. We interpret the reason for the inability to discriminate as follows: In postoperative original learning, rats have to learn to extract the experimenter's defined relevant cues for discrimination from various kinds of potential stimuli, and this might still be difficult, even for OEBs that possess expanded uncrossed visual pathways.

Is there any way to make OEBs and OETs capable of mastering $\mathrm{BW}$ discrimination when learning occurs after the CT visual cortex lesions? We found out that the answer is "yes." During an investigation of the behavioral functions of the expanded uncrossed visual pathways in OEBs, we studied the possibility of neural reorganization in the IP visual cortex, which would be brought out by long-term callosal deafferentation. Specifically, we (Ikeda et al., 1992) examined the effects of callosal transections made at 3 weeks of age and at 13 weeks of age upon the acquisition of BW discrimination using OEBs and OETs. Ten weeks following the callosal transections, rats received the $\mathrm{CT}$ visual cortex le- sions, and after a recovery period of 10 days, the discrimination training was started. The results indicate that (i) OEBs were endowed with the ability to acquire the discrimination habit when the callosal transections were made at 3 weeks of age, and to a lesser extent at 13 weeks of age, and (ii) OETs did not benefit at all, regardless of the age when the callosal transections were made.

These findings were interpreted to show the occurrence of a greater synaptic reorganization in the IP visual cortex of OEBs, which was hypothesized to take place in a manner as follows: (i) there are more neurons receiving callosal afferents in OEBs than in OETs (Cusick \& Lund, 1982, Lund, Chang, \& Land, 1984, Rhoades \& Dellacroce, 1980), and those afferents should degenerate by the 10 -week-old callosal transected lesions, and (ii) there are more thalamic afferents in OEBs than in OETs projecting from the LGNd and the posterior nucleus of the thalamus to the IP visual cortex, and the axon terminals of those afferents would, in turn, proliferate and occupy the synaptic sites vacated by the degenerated callosal afferents. Such neural reorganization is known to be more active in infancy than in adulthood (Hoff, Scheff, \& Cotman, 1982; Lynch \& Gall, 1980; Lynch, Stanfield, \& Cotman, 1973; McWilliams \& Lynch, 1983).

The above findings and speculations led us to undertake the present study. Since the CT visual cortex lesions should result in damage of the callosal neurons projecting to the IP visual cortex, we became curious to know if the effects of 10-week-old CT visual cortex lesions on the acquisition of $\mathrm{BW}$ discrimination were similar to those of the 10-week-old callosal transected lesions. The purpose of the present study was to find an answer to this question.

\section{Method \\ Subjects and Monocular Enucleation}

Fifty one male albino rats of the Wistar strain served as subjects. Dependent upon the time difference of the one eye enucleation, they were divided into two types. One type had the right eye removed within 24 hours after birth (OEB). The other had the right eye enucleated at 81 days of age (nearly 12 weeks of age: OET). Enucleation of the eye was performed under sodium pentobarbital anesthesia $(50 \mathrm{mg} / \mathrm{kg}$, i.p.) in OETs, and under an ether anesthesia in OEBs. The rats were weaned from their mothers at 30 days of age, and thereafter were housed in group cages (two to three rats per 
cage) and were maintained on an ad lib food and water schedule under normal day-night cycles. Each of OEBs and OETs was assigned further into the experiments of 2 conditions (condition A and B). Conditions A and B were as follows.

\section{Condition A}

Fifteen OEBs and 14 OETs served as subjects. They all had the CT visual cortex injured at 3 weeks of age, and 10 weeks later (at 13 weeks of age) were given the training. The mean body weight of the OEB group at the beginning of training was $454.0 \mathrm{~g}$ and that of the OETs was $441.8 \mathrm{~g}$. There was no significant difference between the two groups $\{t(27)=0.602\}$.

\section{Condition B}

Eleven OEBs and 11 OETs served as subjects. They had the CT visual cortex damaged at 13 weeks of age, and 10 weeks later (at 23 weeks of age) were given the training. The mean body weight of the OEB group was $535.5 \mathrm{~g}$ and that of the OET group was $534.5 \mathrm{~g}$. Again, no significant difference was found between the two groups $\{t(20)=0.056\}$.

\section{Cortical Lesion}

All subjects had the right visual cortex removed at either 3 weeks of age or 13 weeks of age. The lesions were performed by aspiration under sodium pentobarbital anesthesia $(50 \mathrm{mg} / \mathrm{kg}$, i.p.). The lesions were intended to extend from the tip of the occipital pole to 6 $\mathrm{mm}$ anteriorly and from the midline to $6 \mathrm{~mm}$ laterally at 3 weeks of age, and from the tip of the occipital pole to $7 \mathrm{~mm}$ anteriorly and from the midline to $7 \mathrm{~mm}$ laterally at 13 weeks of age. The lesions included almost all of areas 17, 18, and 18a as defined by Krieg (1946). Following surgery, the animals were given prophylactic doses of an antibiotic.

\section{Training Apparatus and Procedure}

The training apparatus was a modification of the shock-avoidance discrimination box originally devised by Thompson and Bryant (1955), in which the animals were trained on BW discrimination (black $6.2 \mathrm{~cd} / \mathrm{m}^{2}$, white $202.1 \mathrm{~cd} / \mathrm{m}^{2}$ ) by basically the same procedures as those employed in previous studies (e.g., Ikeda et al., 1983). Briefly, the white door was the positive stimulus, and the electrical shock $(50 \mathrm{~V}, \mathrm{AC})$ was given

from the floor grids. Training consisted of 20 trials a day, and it continued until the animals reached a criterion of three successive blocks of ten trials in which the first and second blocks had more than nine avoidances and the third one had more than eight avoidances (9/10, $9 / 10,8 / 10)$. The training was made within trial limits of
Table 1 Summary of Age for the Treatment Conditions

\begin{tabular}{clrr}
\hline & $\begin{array}{c}\text { Monocular } \\
\text { Enucleation }\end{array}$ & CT Lesion & Training \\
\hline Condition A & & & \\
OEB & at birth & $3 \mathrm{wks}$ & $13 \mathrm{wks}$ \\
OET & nearly $12 \mathrm{wks}$ & $3 \mathrm{wks}$ & $13 \mathrm{wks}$ \\
Condition B & & & \\
OEB & at birth & $13 \mathrm{wks}$ & $23 \mathrm{wks}$ \\
OET & nearly 12 wks & $13 \mathrm{wks}$ & $23 \mathrm{wks}$ \\
\hline
\end{tabular}

a total of 300 trials. Table 1 summarizes the treatments.

\section{Evaluation of Discrimination Acquisition}

The performance of discrimination acquisition was evaluated in terms of the number of errors to the criterion. For statistical analysis of the data, the MannWhitney U-test (two-tailed) was employed.

\section{Histological Procedure}

After the experiment was over, each rat was deeply anesthetized with an overdose of sodium pentobarbital, and was perfused through the heart, using a peristaltic pump, first with $100-150 \mathrm{ml}$ saline at room temperature, followed by $100-150 \mathrm{ml}$ of cold $4 \%$ paraformaldehyde in $0.1 \mathrm{M}$ phosphate buffer (PB; $\mathrm{pH}$ 7.4). The brain was removed from the skull and postfixed with the same fixative solution at $4{ }^{\circ} \mathrm{C}$ overnight, and was then cryoprotected in $0.1 \mathrm{M} \mathrm{PB}$ containing 10,15 and $20 \%$ sucrose with $0.1 \% \mathrm{NaN}$ (subscript: 3 ) at $4^{\circ} \mathrm{C}$ until it sank, each for an overnight period, respectively. After the dorsal surface of the brain was photographed by a Medical Nikkor camera, coronal $30 \mu \mathrm{m}$ sections were cut in a cryostat $\left(-18^{\circ} \mathrm{C}\right)$ throughout the LGNd, and every 5 th section was processed with Nissl stain. Subsequently, the amount of retrograde degeneration, as defined by a complete neuronal cell loss in the rostral, middle, and caudal portions of the LGNd, was assessed under a microscope for all animals. Furthermore, using a photograph of the dorsal surface of the brain (Fig. 1 and 2; top, left) and a low power photomicrograph of the coronal section of the LGNd (Fig. 1 and 2; middle), the extent of the CT cortical lesions and the size of the IP and CT LGNd were measured for each rat by Mac NIH Image. They were quantified by tracing the circumference of the destroyed cortical area and that of the LGNd. 

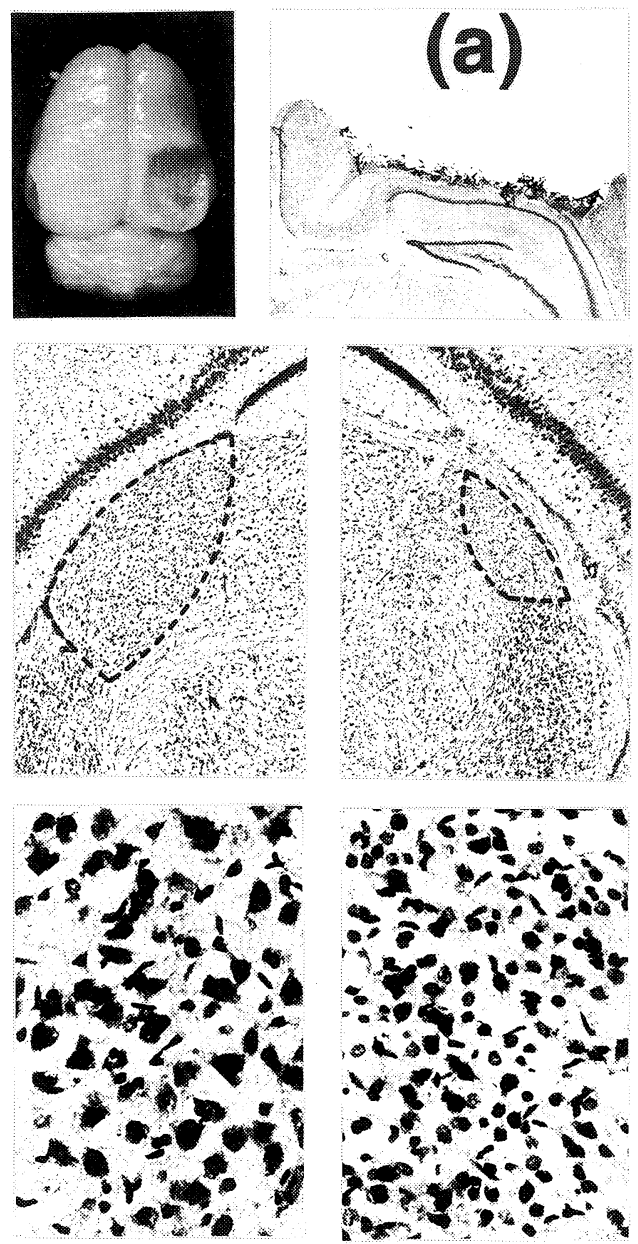

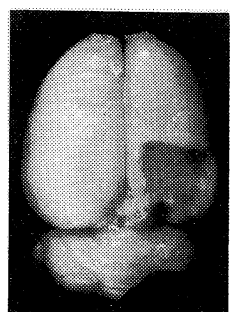

(b)
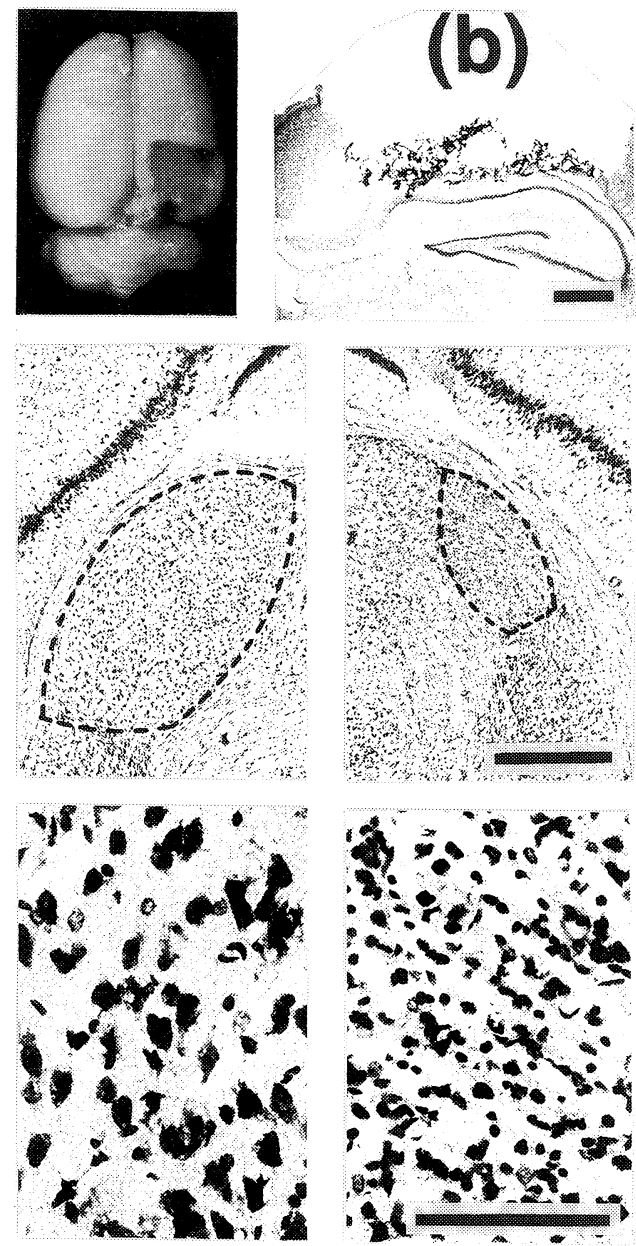

Fig. 1 Photographs of the dorsal surface of the brain (top, left), and photomicrographs of coronal sections through the CT cortical lesion site (top, right) and the LGNds ipsilateral (left) and contralateral (right) to the remaining eye under low (middle) and high (bottom) magnifications. (a) Rat \#7 of the OEB group in condition A. (b) Rat \#6 of the OET group in condition A. Both of the contralateral LGNds in the figure show an almost complete neuronal cell loss. Note (1) that the contralateral LGNd in both (a) and (b) shows marked shrinkage due to visual cortical lesions of the same hemisphere at 3 weeks of age, and (2) that the ipsilateral LGNd in (a) shows shrinkage due to monocular enucleation at birth, whereas such shrinkage was not observed in the ipsilateral LGNd in (b), in which one eye was removed after maturity. Dashed outline indicates the circumference of the LGNd. Bars: Top-right, $1 \mathrm{~mm}$; Middle, $500 \mu \mathrm{m}$; Bottom, $100 \mu \mathrm{m}$.

\section{Results}

\section{Histological Results}

Figures 1 and 2 show the dorsal surface of the brain, the coronal section through the $\mathrm{CT}$ cortical lesion site and the LGNd in the IP and CT hemispheres of four representative animals: one for each of the OEB and OET groups in condition A (Fig.1), and that in condition B (Fig. 2), respectively.

Cortical Lesions. In all subjects damage was restricted to the cortex and did not extend to the subcortical areas as seen in Fig. 1 and 2 (top, right). Although posterior cortical lesions were large enough to include areas 17, 18, and 18a in most of subjects, some cortical sparings were observed along the midline in all subjects (Fig. 1 and 2; top, left). Comparisons of the cortical lesion size with the rate of learning showed no systematic relations either in OEBs or in OETs, for either condition A or condition B. Also, none of the subjects showed apparent motor deficits as a result of the large extent of the cortical lesions.

Retrograde Degeneration of the Contralateral $L G N d$. Although retrograde degeneration of the LGNd 

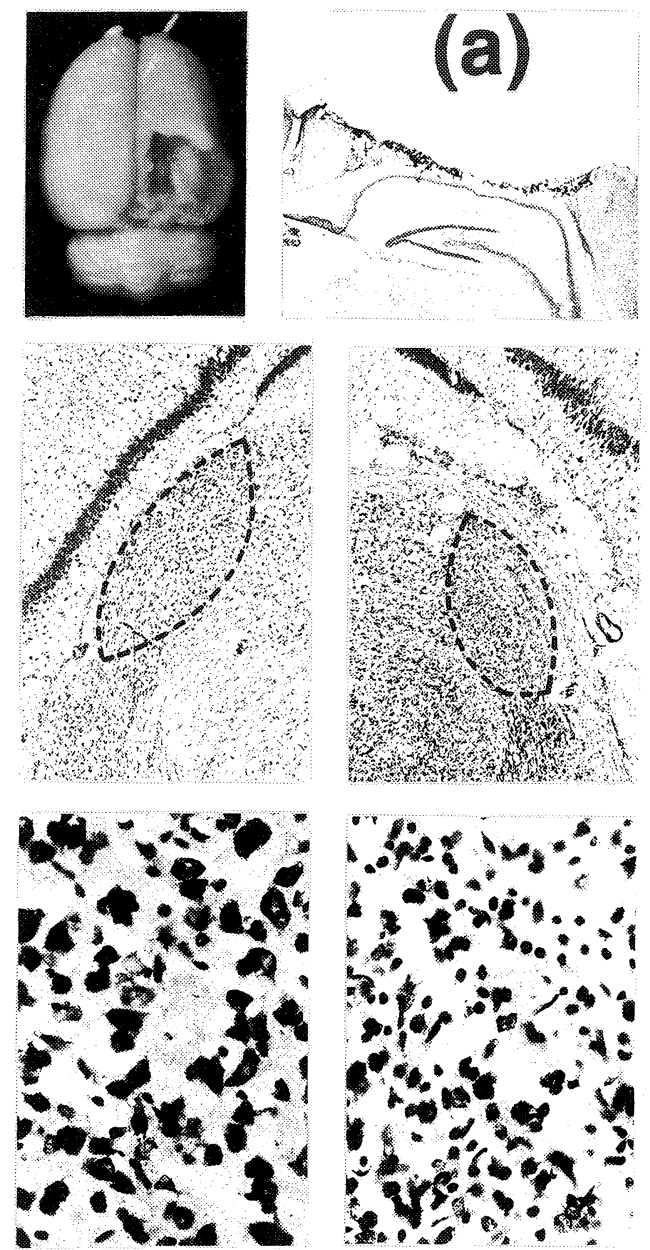

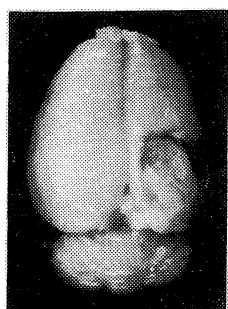

(b)
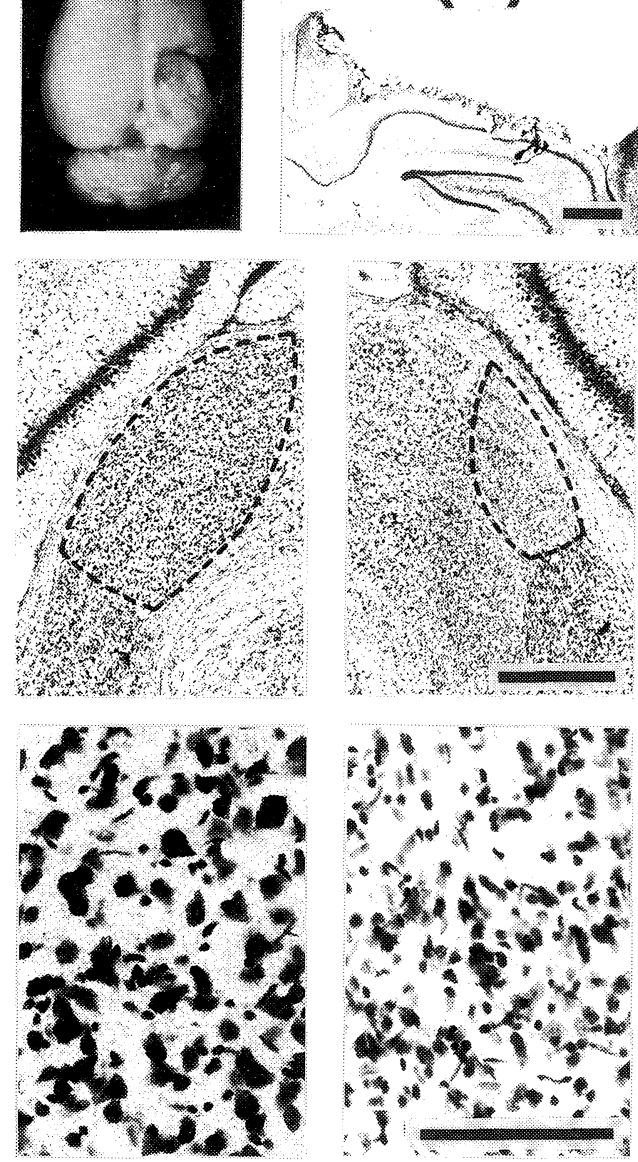

Fig. 2 Photographs of the dorsal surface of the brain (top, left), and photomicrographs of coronal sections through the CT cortical lesion site (top, right) and the LGNds ipsilateral (left) and contralateral (right) to the remaining eye under low (middle) and high (bottom) magnifications. (a) Rat \#2 of the OEB group in condition B. (b) Rat \#4 of the OET group in condition B. Both of the contralateral LGNds in the figure show an almost complete neuronal cell loss. Note (1) that the contralateral LGNd in both (a) and (b) shows marked shrinkage due to visual cortical lesions of the same hemisphere at 13 weeks of age, and (2) that the ipsilateral LGNd in (a) shows shrinkage due to monocular enucleation at birth, whereas such shrinkage was not observed in the ipsilateral LGNd in (b), in which one eye was removed after maturity. Dashed outline indicates the circumference of the LGNd. Bars: Top-right, $1 \mathrm{~mm}$; Middle, $500 \mu \mathrm{m}$; Bottom, $100 \mu \mathrm{m}$.

neuronal cells contralateral to the remaining eye as a result of the cortical lesions in the same hemisphere was almost complete in most of the subjects (Fig. 1 and 2; bottom, right), in one OEB, \#2, and two OETs, \#5 and $\# 8$, for condition $\mathrm{A}$, and in one OEB, \#4, and one OET, $\# 1$, for condition B, some healthy cells were found to be scattered in the LGNd. However, there was no consistent relation in the performance of discrimination between rats with neuronal sparings of the LGNd and those with complete degeneration.

Shrinkage of the Contralateral LGNd. Due to the cortical lesion, the size of the LGNd contralateral to the remaining eye was found to be reduced to $16-22 \%$ as small as the normal size in condition A (Fig. 1; middle, right), and 34-41\% in condition B (Fig. 2; middle, right).

Shrinkage of the Ipsilateral LGNd. The LGNd ipsilateral to the remaining eye of the OEB group in both conditions A and B showed shrinkage, $41-57 \%$ as small as the normal size, as a result of monocular enucleation at birth (Fig. 1 (a) and 2 (a); middle, left), whereas such shrinkage was not observed in the LGNd of the OET group in both conditions $\mathrm{A}$ and $\mathrm{B}$, in which one eye was 
Table 2 Numbers of Errors Made by Individual Subjects in Acquisition of Discrimination

\begin{tabular}{|c|c|c|c|c|c|c|c|}
\hline \multicolumn{4}{|c|}{ Condition A } & \multicolumn{4}{|c|}{ Condition B } \\
\hline \multirow[t]{2}{*}{$\begin{array}{l}\text { Subject } \\
\text { No. }\end{array}$} & \multirow[t]{2}{*}{$\begin{array}{l}\text { Errors to } \\
\text { Criterion in } \\
\text { Acquisition }\end{array}$} & \multicolumn{2}{|c|}{$\begin{array}{l}\text { Percetnt Correct Re- } \\
\text { sponses in the First and } \\
\text { Last } 20 \text { Trials After Ad- } \\
\text { ditional IP Lesion }\end{array}$} & \multirow[t]{2}{*}{$\begin{array}{l}\text { Subject } \\
\text { No. }\end{array}$} & \multirow[t]{2}{*}{$\begin{array}{l}\text { Errors to } \\
\text { Criterion in } \\
\text { Acquisition }\end{array}$} & \multicolumn{2}{|c|}{$\begin{array}{l}\text { Percetnt Correct } \mathrm{Re}- \\
\text { sponses in the First and } \\
\text { Last } 20 \text { Trials After Ad- } \\
\text { ditional IP Lesion }\end{array}$} \\
\hline & & First & Last & & & First & Last \\
\hline OEB & & & & OEB & & & \\
\hline 1 & 94 & & & 1 & 96 & & \\
\hline 2 & + & & & 2 & 95 & & \\
\hline 3 & 41 & & & 3 & 68 & & \\
\hline 4 & 37 & & & 4 & 97 & 80 & 60 \\
\hline 5 & 43 & & & 5 & + & & \\
\hline 6 & 87 & & & 6 & 118 & 50 & 50 \\
\hline 7 & 62 & & & 7 & 66 & 65 & 50 \\
\hline 8 & 60 & & & 8 & 104 & & \\
\hline 9 & 44 & & & 9 & + & & \\
\hline 10 & 72 & 70 & 45 & 10 & 74 & 50 & 30 \\
\hline 11 & 62 & & & 11 & 60 & 50 & 25 \\
\hline 12 & 86 & & & mdn & 96 & & \\
\hline 13 & 127 & 50 & 55 & & & & \\
\hline 14 & 55 & 50 & 35 & & & & \\
\hline 15 & 49 & 60 & 45 & & & & \\
\hline mdn & 62 & & & & & & \\
\hline OET & & & & OET & & & \\
\hline 1 & + & & & 1 & + & & \\
\hline 2 & + & & & 2 & + & & \\
\hline 3 & 96 & & & 3 & 66 & 50 & 50 \\
\hline 4 & 61 & & & 4 & + & & \\
\hline 5 & 65 & & & 5 & + & & \\
\hline 6 & 78 & & & 6 & + & & \\
\hline 7 & 80 & & & 7 & 67 & 70 & 75 \\
\hline 8 & 46 & & & 8 & + & & \\
\hline 9 & 77 & & & 9 & + & & \\
\hline 10 & 49 & 50 & 60 & 10 & + & & \\
\hline 11 & + & & & 11 & + & & \\
\hline 12 & 37 & 60 & 75 & mdn & + & & \\
\hline 13 & 68 & 70 & 70 & & & & \\
\hline 14 & 87 & 60 & 70 & & & & \\
\hline mdn & 77.5 & & & & & & \\
\hline
\end{tabular}

removed after maturity (Fig. 1 (b) and 2 (b); middle, left).

\section{Behavioral Results}

Table 2 shows the numbers of errors made by the individual subjects of OEBs and OETs to reach criterion in condition $\mathrm{A}$ and $\mathrm{B}$. Table 3 shows the data of the previous study in the corresponding conditions. As seen from Tables 2 and 3, the overall pattern of the results of the present study was basically the same as that of the previous study. However, statistical analyses revealed slight differences. In the present study there is no reliable difference in the numbers of errors to reach criterion between OEBs $(\operatorname{mdn}=62)$ and OETs $(\operatorname{mdn}=77.5)$ in condition $\mathrm{A}$, showing both OEBs and OETs were able to acquire the discrimination equally well $(U(15,14)=$ 78). In condition $B$, while no significant difference was obtained in the numbers of errors to criterion between 
Table 3 Numbers of Errors Made by Individual Subjects in Acquisition of Discrimination in the Previous Study (Ikeda et al., 1992)

\begin{tabular}{|c|c|c|c|c|}
\hline & \multicolumn{2}{|c|}{ Condition A } & \multicolumn{2}{|c|}{ Condition B } \\
\hline \multirow[t]{2}{*}{$\begin{array}{l}\text { Subject } \\
\text { No. }\end{array}$} & \multicolumn{2}{|c|}{$\begin{array}{l}\text { Errors to } \\
\text { Criterion in } \\
\text { Acquisition }\end{array}$} & \multicolumn{2}{|c|}{$\begin{array}{l}\text { Errors to } \\
\text { Criterion in } \\
\text { Acquisition }\end{array}$} \\
\hline & OEB & OET & OEB & OET \\
\hline 1 & 104 & 83 & 75 & + \\
\hline 2 & 86 & + & + & + \\
\hline 3 & + & 66 & + & 86 \\
\hline 4 & 94 & + & 104 & + \\
\hline 5 & 48 & + & 113 & + \\
\hline 6 & 133 & + & 66 & + \\
\hline 7 & 40 & 121 & + & 124 \\
\hline 8 & + & 81 & 109 & + \\
\hline 9 & + & 112 & + & \\
\hline 10 & 51 & + & 93 & \\
\hline 11 & 116 & 97 & + & \\
\hline 12 & 63 & + & 71 & \\
\hline 13 & 71 & 85 & & \\
\hline 14 & 56 & + & & \\
\hline 15 & 62 & + & & \\
\hline 16 & & + & & \\
\hline mdn & 86 & + & 111 & + \\
\hline
\end{tabular}

$+=$ Rats that failed to reach criterion within 300 trials.

OEBs and OETs in the previous study, OEBs $(\mathrm{mdn}=$ 96) could master the discrimination significantly faster than OETs $((\operatorname{med}=+)(U(11,11)=27.5) p<0.04)$ in the present study.

Also, in both OEBs and OETs the acquisition rate of $\mathrm{BW}$ discrimination was faster when the $\mathrm{CT}$ visual cortex lesions were made at 3 weeks of age than at 13 weeks of age $(U(15,11)=36.5, p<0.02$ for OEBs, $U$ $(14,11)=31.5, p<0.02$ for OETs $)$.

In order to compare the degree of the facilitative effects on the acquisition of BW discrimination between the CT visual cortex lesions and the callosal transected lesions in OEBs and OETs, all OEB and OET data in the present study (Table 2) were pooled regardless of the age of the CT visual cortex lesions. Similarly, data of the previous study were pooled regardless of the age of the callosal transected lesions (Table 3). It was found that the acquisition of $\mathrm{BW}$ discrimination was reliably facilitated more in the CT visual cortex lesions than in the callosal transected lesions ( $m d n=86$ for the $\mathrm{CT}$ visual cortex lesions; $\mathrm{mdn}=121$ for the callosal transected lesions; $\mathrm{CR}(51,51)=2.47, p<0.02)$.

The role of the IP visual cortex in mediating learn- ing of the discrimination was examined in the present study; 15 subjects (4 OEBs and 4 OETs in condition A; 5 OEBs and 2 OETs in condition B) were arbitrarily picked up from those OEBs and OETs that reached criterion, and were tested for the discrimination capacity following the additional lesions of the IP visual cortex. Performance of the discrimination was evaluated in terms of percent correct responses in the first and last 20 trials in the stipulated 100 relearning trials (Table 2). The median percent correct responses for the first and last 20 trials are 60 and 50, respectively, indicating that the IP visual cortex plays an essential role in BW discrimination.

\section{Discussion}

The present study indicates that the overall pattern of the results is in general the same as that of the previous study; when the CT visual cortex was extirpated at 3 weeks of age (Condition A), not only OEBs but also OETs mastered the task. The present study also shows that when the time of the CT visual cortex lesions was delayed until 13 weeks of age (Condition B), OEBs could still master the task, but OETs could no longer do it. The result that is different from those of the previous study is that OETs in condition A became able to acquire the discrimination habit, whereas they did not in the previous study. This difference, however, should not be taken as a sign of qualitative change of neural reorganization in OETs between the present and the previous studies, because 7 out of 16 OETs $(43.8 \%)$ learned the task in the previous study.

Since the additional lesions of the IP visual cortex after the completion of the discrimination made OEBs and OETs unable to restore the habit, the IP visual cortex is assumed to play an essential role in mediating BW discrimination. This finding also is accord with that of the previous study.

And now we know that the acquisition of BW discrimination is more facilitated in the present study than in the previous study. In other words, the long-term CT visual cortex lesions facilitate the acquisition of $\mathrm{BW}$ discrimination much more than the long-term callosal transected lesions.

Then, a question is raised as to how a further facilitation in the learning ability of the IP visual cortex as observed in the present study would be explained. Would it be possible to extend the hypothesis (see Introduction) to explain it? The answer appears to be "yes."

A number of investigations have suggested that terminal proliferation or reactive synaptogenesis is trig- 
gered and facilitated by neurotrophic factors, released either by glial cells or neurons in the course of terminal degeneration (e.g., Cotman \& Nadler, 1978; Manthorpe, Nieto-Sampedro, Skaper, Lewis, Barbin, Longo, Cotman, \& Varon, 1983). The degree of reactive synaptogenesis is influenced by many factors, such as the amount of released neurotrophic factors, age, the primed state of terminals to sprout, etc. When only the callosal transected lesions are made in the previous study, it is assumed that a main releasing source of neurotrophic factors is around the terminal degeneration site of the callosal fibers, where reactive synaptogenesis takes place. But when the CT posterior cortex is largely damaged, in neonatal rats as well as in adult rats, it has been reported (i) that the damaged cortical site itself releases neurotrophic factors, which then spread over the IP cortex through the ventricular system, and (ii) that the level of neurotrophic activity is roughly proportional to the volume of the damaged cavity in the cortex (Manthorpe et al., 1983; Nieto-Sampedro \& Cotman, 1985; NietoSampedro, Manthorpe, Barbin, Varon, \& Cotman, 1983). And it is also known that neural reorganization or reactive synaptogenesis is more active in infancy than in adulthood (Hoff et al., 1982; Lynch \& Gall, 1980; Lynch et al., 1973; McWilliams \& Lynch, 1983). Therefore, it is conceivable that by this added amount of released neurotrophic factors resulting from the $\mathrm{CT}$ visual cortex, a further facilitation of neural reorganization or reactive synaptogenesis in the IP visual cortex is expected to occur in the present study, and consequently, it serves to enhance the acquisition ability of BW discrimination not only in OEBs but also in OETs.

Thus, the following point is implicated in the present study. If the amount of trophic factors is sufficient for reactive synaptogenesis, then, the attainment of level of neural reorganization sufficient for the acquisition of BW discrimination would take place through the degeneration of those callosal terminals. This is not only so in the OEBs that are endowed with exuberant callosal terminals in the IP visual cortex, but also in the OETs that have regular numbers of callosal terminals and thalamic afferents.

As another contributing factor, on the other hand, one may consider the increased use of the uncrossed visual pathways to visually explore the surroundings. Recent studies on the adult rat somatosensory cortex (Jones, Kleim, \& Greenough, 1996; Jones \& Schallert, 1992; Jones \& Schallert, 1994) have demonstrated that the increase in the number of synapses, the volume of synaptic process, and the amount of synaptic membrane per neuron of layer $\mathrm{V}$ of the cortex are brought about by damage to the contralateral homotopic cortical area, but only if use of the unaffected forelimb is allowed. Therefore, it can be speculated that the increased use of the uncrossed visual pathways might also result in morphological changes in the IP visual cortex, which would eventually facilitate the acquisition of the BW discrimination.

So there is the possibility that an interaction between the increase in trophic factors and the increased use of the uncrossed visual pathways may lead to the ultimate enhancement of the acquisition ability of BW discrimination in the present study. The relative contributions of these two factors can probably be evaluated by finding the answer to the following question: Can the IP visual cortex of OEBs and OETs that have the CT cortex (other than the visual cortex) lesioned, and also have the corpus callosum transected at either 3 or 13 weeks of age, possess the same acquisition ability of BW discrimination, when the ability is tested 10 weeks following those lesions? The separation of these two factors is of great interest, but remains to be analyzed.

\section{REFERENCES}

Chan, S. O., \& Jen, L. S. 1988 Enlargement of uncrossed retinal projections in the albino rat: Additive effects of neonatal eye removal and thalamectomy. Brain Research, 461, 163168.

Cotman, C. W., \& Nadler, J. V. 1978 Reactive synaptogenesis in the hippocampus. In C. W. Cotman (Ed.), Neuronal plasticity. New York: Raven Press. Pp. 227-272.

Cusick, C. G., \& Lund, R. D. 1982 Modification of visual callosal projections in rats. Journal of Comparative Neurology, 212, 385-398.

Hoff, S. F., Scheff, S. W., \& Cotman, C. W. 1982 Lesioninduced synaptogenesis in the dentate gyrus of aged rats: II. Demonstration of an impaired degeneration clearing response. Journal of Comparative Neurology, 205, 253-259.

Ikeda, Y., Sakai, M., \& Yagi, F. 1988 Effects of superior colliculus lesion upon a black-white discrimination learning in the albino rat with one eye removed at birth. Physiology \& Behavior, 43, 657-663.

Ikeda, Y., Sakai, M., \& Yagi, F. 1992 Long-term callosal lesions and learning of a black-white discrimination by oneeyed rats. Physiology \& Behavior, 52, 851-858.

Ikeda, Y., Yagi, F., \& Sakai, M. 1983 The role of crossed and uncrossed optic pathways mediating black-white discrimination in rats with one eye enucleated at birth. Physiology \& Behavior, 30, 885-890.

Jeffery, G. 1985 Early unilateral eye removal produces a regional gradient in soma sizes in the uncrossed projection in 
the rat. Developmental Brain Research, 19, 155-159.

Jen, L. S., \& Lund, R. D. 1981 Experimentally induced enlargement of the uncrossed retinotectal pathway in rats. Brain Research, 211, 37-57.

Jones, T. A., Kleim, J. A., \& Greenough, W. T. 1996 Synaptogenesis and dendritic growth in the cortex opposite unilateral sensorimotor cortex damage in adult rats: a quantitative electron microscopic examination. Brain Research, 733, 142-148.

Jones, T. A., \& Schallert, T. 1992 Overgrowth and pruning of dendrites in adult rats recovering from neocortical damage. Brain Research, 581, 156-160.

Jones, T. A., \& Schallert, T. 1994 Use-dependent growth of pyramidal neurons after neocortical damage. The Journal of Neuroscience, 14, 2140-2152.

Krieg, W. J. S. 1946 Connections of the cerebral cortex. I. The albino rat. A. Topography of the cortical areas. Journal of Comparative Neurology, 84, 221-275.

Laemle, L. K., \& Labriola, A. R. 1982 Retinocollicular projections in the neonatal rat: An anatomical basis for plasticity. Developmental Brain Research, 3, 317-322.

Lund, R. D., Chang, F. L. F., \& Land, P. W. 1984 The development of callosal projections in normal and one-eyed rats. Developmental Brain Research, 14, 139-142.

Lund, R. D., Cunningham, T. J., \& Lund, J. S. 1973 Modified optic projections after unilateral eye removal in young rats. Brain, Behavior and Evolution, 8, 51-72.

Lynch, G., \& Gall, C. 1980 The regulation of fiber growth and synaptogenesis in the developing hippocampus. Current Topics in Developmental Biology, 15, 159-180.

Lynch, G., Stanfield, B., \& Cotman, C. W. 1973 Developmental differences in post-lesion axonal growth in the hippocampus. Brain Research, 59, 155-168.

Manthorpe, M., Nieto-Sampedro, M., Skaper, S. D., Lewis, E. R., Barbin, G., Longo, F. M., Cotman, C. W., \& Varon, S. 1983 Neurotrophic activity in brain wounds of the developing rat. Correlation with implant survival in the wound cavity. Brain Research, 267, 47-56.
McWilliams, R., \& Lynch, G. 1983 Rate of synaptic replacement in denervated rat hippocampus declines precipitously from the juvenile period to adulthood. Science, 221, 572574.

Nieto-Sampedro, M., \& Cotman, C. W. 1985 Growth factor induction and temporal order in central nervous system repair. In C. W. Cotman (Ed.), Synaptic plasticity. New York: Guilford Press. Pp. 407-456.

Nieto-Sampedro, M., Manthorpe, M., Barbin, G., Varon, S., \& Cotman, C. W. 1983 Injury-induced neurotrophic activity in adult rat brain: Correlation with survival of delayed implants in the wound. The Journal of Neuroscience, 3 , 2219-2229.

Rhoades, R. W., \& Dellacroce, D. D. 1980 Neonatal enucleation induces an asymmetric pattern of visual callosal connections in hamsters. Brain Research, 202, 189-195.

Sakai, M., Ikeda, Y., \& Yagi, F. 1991 Differential effects of unilateral optic tract transections and visual cortical lesions upon a pattern discrimination in albino rats. Physiology \& Behavior, 50, 409-416.

Sakai, M., Ikeda, Y., \& Yagi, F. 1996 Discrimination performance and resolution capacity of uncrossed visual pathways in one-eyed albino rats. Physiology \& Behavior, 59, 141146.

Sefton, A. J., \& Lam, K. 1984 Quantitative and morphological studies on developing optic axons in normal and enucleated albino rats. Experimental Brain Research, 57, 107-117.

Thompson, R., \& Bryant, J. H. 1955 Memory as affected by activity of the relevant receptor. Psychological Reports, 1 , 393-400.

Yagi, F., \& Sakai, M. 1979 Effect of one eye removal at birth on retention of a visual habit in the albino rat. Japanese Psychological Research, 21, 181-188.

Yagi, F., Sakai, M., \& Ikeda, Y. 1989 Effects of delayed monocular enucleation after birth upon black-white discrimination learning in the albino rat. Physiology \& Behavior, 45, 1011-1015. 